\title{
目标搜索与识别的视觉注意网络与学习方法*
}

\author{
吕杰罗芳颖 袁泽剑 \\ (西安交通大学电子与信息工程学院 西安 710049)
}

\begin{abstract}
摘要: 提出一种循环视觉注意网络来同时进行目标搜索和识别。该网络能够从图像中自动选择一个局部观测序列, 通过融合 局部详细表观和粗略上下文视觉信息, 实现视觉目标的高精度定位与识别, 比传统的滑动窗口和全图卷积的方法具有更高的 目标搜索效率。此外, 提出了一种混合损失函数来对网络参数进行端到端的多任务学习, 特别在视觉注视点序列损失函数中 引入随机性和目标启发的组合策略, 可以有效地挖掘更丰富的上下文信息, 保证注意点快速接近视觉目标。建立了一个真实 场景数据集来验证该模型在感兴趣目标和小目标搜索与识别的性能。试验结果表明, 该方法通过几个注视点转移, 就能够在 一幅图像上预测一个视觉目标的准确边框, 并在大图像上获得比较高的搜索速度。开放源代码用于该方法验证与比较分析。 关键词: 注意力模型; 强化学习; 目标检测; 注意策略
\end{abstract} 中图分类号: TG156

\section{Visual Attentional Network and Learning Method for Object Search and Recognition}

\author{
LÜ Jie LUO Fangying YUAN Zejian
}

(School of Electronic and Information Engineering, Xi'an Jiaotong University, Xi'an 710049)

\begin{abstract}
A recurrent visual network is proposed to search and recognize an object simultaneously. The network can automatically select a sequence of local observations, and accurately localize and recognize objects by fusing those local detail appearance and rough context visual information. The method is more efficient than other methods with sliding windows or convolution on a whole image. Besides, a hybrid loss function is proposed to learn parameters of the multi-task network end-to-end. Especially, The combination of stochastic and object-awareness strategy is imported into visual fixation loss, which is beneficial to mine more abundant context and ensure fixation point close to object as fast as possible. A real-world dataset is built to verify the capacity of the method in searching and recognizing the object of interest including those small ones. Experiments illustrate that the method can predict an accurate bounding box for a visual object, and achieve higher searching speed. The source code will be opened to verify and analyze the method.
\end{abstract}

Key words: attentional model; reinforcement learning; object detection; fixation strategy

\section{0 前言}

在机器人与环境的交互中, 对视觉目标的搜索 结果是直接对决策行为产生影响的, 一种高效准确 的视觉搜索模型能显著提高机器人与环境的交互性 能。然而, 设计一种同时满足高效和准确的视觉目 标搜索识别模型是颇具挑战性的。大多数传统的目 标检测方法, 比如级联检测器 ${ }^{[1]}$ 和 $\mathrm{DPM}^{[2-3]}$, 采取了 滑动窗口的策略, 这意味着检测器需要遍历整幅图 像的每个可能的位置。由于计算资源在显著性区域

* 国家自然科学基金 $(91648121,61573280$ ) 和国家重点研究计划 (2016YFB001001)资助项目。20180620 收到初稿, 20190319 收到修改稿
和大部分的背景区域不合理分配，这些方法显然是 不高效的。近年来, 神经网络的方法在目标检测 的领域已经取得了巨大的成功。基于区域推荐的 方法 ${ }^{[4-6]}$ 首先获得大量疑似目标的候选窗口, 然后基 于候选窗口预测目标边框。但是过多的候选区域导 致检测器变慢。而且检测的精度受限于区域推荐算 法的性能。单发方式 ${ }^{[7-8]}$ 的目标检测由于采用直接回 归和网格策略, 已经取得最好的速度和精度。然而, 这种方法对于检测小目标和长宽比不太正常的目标 是有困难的。

人类采用扫视和凝视多步观看的方式来搜索和 识别目标, 每一步只观看详细的小区域和粗略的上 下文信息 ${ }^{[9]}$, 而不是规则的扫描。在真实场景下, 上 
下文信息对于定位和识别目标而言是重要的 ${ }^{[10-11]}$ 。 特别地, 小目标由于缺乏足够的表观信息, 更加依 赖上下文信息 ${ }^{[12]}$, 比如在高速公路上的前方远处的 车辆可以根据天空、地面、车道线以及其他车辆进 行定位。多步注意策略是一种建模目标与环境之间 的关系的灵活有效的方式。

本文因此提出了一种循环注意的定位识别网络 (Recurrent attention to localization and recognition network, RA-LRN), 如图 1 所示。该网络在每个循 环内有三个子网络模块组成: 中央凹描述子提取 器(Fovea descriptor extractor, FDE), 信息融合网络 (Information fusion network, IFN) 和多任务动作

(Multi-task actions, MTA)。给定一个输入图像和注 意点, FDE 模拟视网膜获取多尺度的包含精细小 区域和粗略上下文信息的局部区域，随后由一组 卷积神经网络(Convolutional neural network, CNN) 学习器模仿大脑 V1 区来提取有效的表观特征。在 IFN 中, 全连接网络被用于进行表观和注意点位置 信息融合，一个 3 层的 LSTMs 网络用于仿制大脑 皮层的记忆功能进行多个局部观测的融合，并产 生一个综合的描述矢量。MTA 接收该矢量, 然后 执行三个动作：定位、分类和预测下一个注意点。 这些动作是由全连接层和一些限制数值范围的操 作完成。

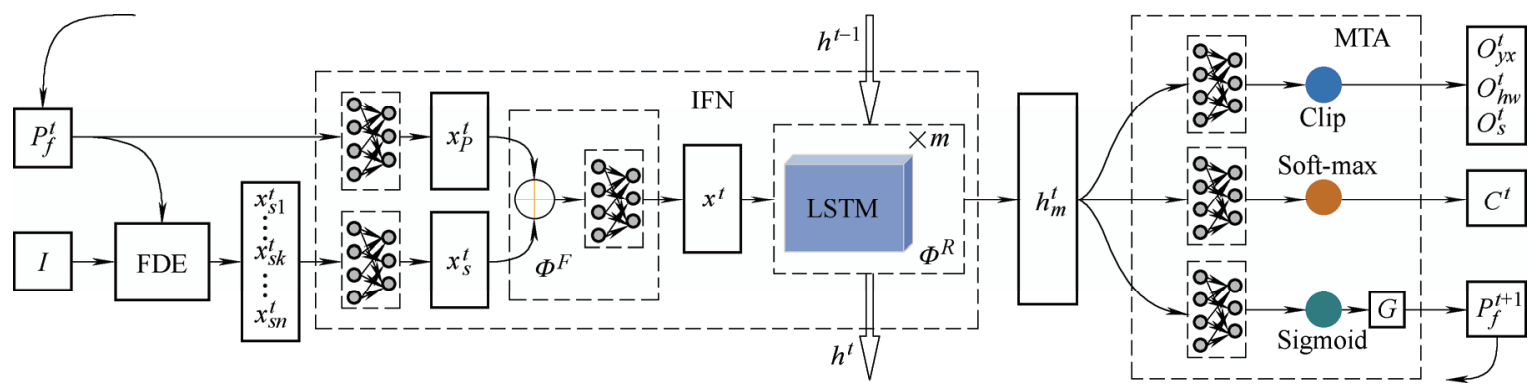

图 1 循环注意力的目标定位识别网络(RA-LRN)整体结构图

在训练时, 本文提出多任务损失函数来对网络 参数进行端到端的训练, 同时还结合随机和目标启 发(SA)策略来对注意点预测进行训练。随机策略(S) 是从一个分布中随机产生下一个注意点, 该方法可 以扩大样本的多样性, 并且在策略-奖励方法的指导 下尽快给出较好的结果。目标启发策略(A)是指最后 一步注意点在 L2 准则下尽量靠近目标的中心，这 对于提高优化和预测的稳定性是有益的。此外本文 还创建了一个真实场景的数据集 FCAR。该数据集 提供了前车的位置信息, 可以用于训练和评估感兴 趣的目标检测任务。

\section{1 相关工作}

在目标检测的任务中, 深度学习的方法比较流 行, 并且近来取得了巨大的成功。为了避免使用滑 动窗口, GIRSHICK 等提出了选择显著性区域然后 再用 $\mathrm{CNN}$ 来进行分类的方法 ${ }^{[4]}$, 随后这类方法又被 改进了精度和速度 ${ }^{[5-6,13]}$ 。尽管如此, 由于选择的区 域众多导致速度仍然很慢, 并且其精度受限于区域 选择算法的性能。单发的模型采用回归和网格化的 策略直接预测目标的位置和类别, 这类方法有更快 的速度和更高的精度 ${ }^{[7-8]}$ 。但是由于没能利用好上下 文的信息，导致对小目标的检测有一定困难。

在神经计算和认知科学中, 上下文对于定位和
识别目标的意义已经被研究 ${ }^{[10-11]}$ 。BELL 等发现上 下文和多尺度描述子能够改善对小目标的检测。为 了将计算资源合理的利用在有效的上下文信息提取 中, LAROCHELLE 等 ${ }^{[14]}$ 提出了类中央凹的图像提 取器, 该方法可以模拟中央凹的功能, 但是太过复 杂而不实用。此外，随机的隐变量也被用于神经网 络以模拟人类注意力策略 ${ }^{[15-16]}$ 。还有研究者采用强 化学习的方法来优化带有隐变量的神经网络 ${ }^{[17]}$ 。

更复杂的循环网络被用来模拟人类的注意力机 制 ${ }^{[18]}$ 。还有一些研究者采用循环或者多级网络来学 习注意力位置 ${ }^{[19-22]}$ 。MNIH 等 ${ }^{[17]}$ 提出循环注意力网 络 RAM 和随机注意策略。GREGOS 等 ${ }^{[23]}$ 提出深度 循环注意写操作 DRAW 并学习样本的隐分布, 然后 应用于分类任务。但是，上述方法都没有采用循环 的结构联合进行定位和识别任务, 随机和目标启发 策略的混合损失函数也没有被讨论。CAICEDO 等 ${ }^{[24]}$ 采用强化学习的方法优化 9 个动作的网络来进行目 标定位, 但是频繁的大小转换导致太多的形变和位 置的不精确。频繁处理大图像也会导致速度缓慢。

\section{2 循环注意力视觉目标搜索与识别}

本文提出了一种根据一些相关的局部观测来同 时预测目标的边框和类别的循环网络 RA-LRN。该 方法的主要模块包括: 中央凹描述子提取器(FDE)、 
信息融合网络(IFN)和多任务动作(MTA)。

\section{1 中央凹描述子提取器}

对于在真实场景中搜索目标而言, 丰富的相关 上下文信息是非常重要的 ${ }^{[11]}$ 。采用模拟视网膜和 V1 区的方式, 本文将类视网膜捕获器和 $\mathrm{CNN}$ 学习器 联合在一起, 这样就能够提取包含丰富上下文信息 和重要空间信息的特征描述子。该网络中的中央凹 描述子提取器如图 2 所示。

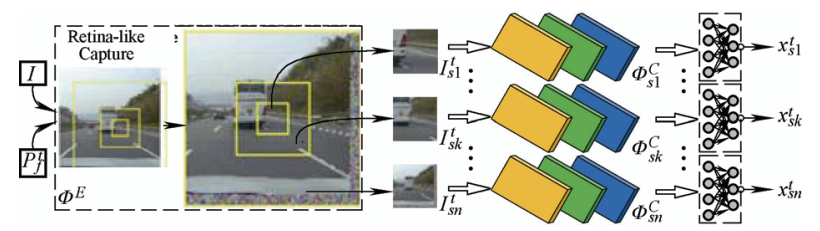

图 2 中央凹描述子提取器的结构图

类视网膜捕获器：类视网膜捕获器 ${ }^{[9]} \boldsymbol{\Phi}^{\mathrm{E}}$ 是本 网络的第一个模块, 控制着表观信息流的输入源。 参数为 $\boldsymbol{\theta}_{\mathrm{E}}$ 该模块在第 $t$ 次循环时以给定的注意点 $P_{f}^{t}$ 为中心从全图 $I$ 中提取若干个不同尺度的图像 块 $I_{s k}^{t}, k=1,2, \cdots, n$ 。设定尺度的个数为 $n$, 最小尺度 大小为 $s_{0}$, 以及尺度因子 $s_{f}$, 捕获器以大小为 $s_{k}=s_{0} \times s_{f}^{k-1}$ 截取第 $k$ 个图像块, 然后将所有的图像 块转换到统一的大小 $s_{u}$ 。数学上, 该映射 $\boldsymbol{\Phi}^{\mathrm{E}}$ 表示 如下

$$
\left\{I_{s k}^{t}\right\}=\boldsymbol{\Phi}^{\mathrm{E}}\left(I, P_{f}^{t}, \boldsymbol{\theta}_{\mathrm{E}}\right) k=1,2, \cdots, n
$$

式中, $\boldsymbol{\theta}_{\mathrm{E}}=\left\{s_{0}, n, s_{f}, s_{u}\right\}$ 是根据少量关于输入图像大 小的先验由人工设置的一组参数。循环网络第一步 的注意点是根据均匀分布采样得到的。

CNN 学习器: 一组 CNN 学习器分别被用于这 些多尺度的图像块 $\left\{I_{s k}^{t}\right\}, k=1,2, \cdots, n$, 来提取有效的 特征。每个 CNN 学习器由三个参数分别为 $\theta_{\mathrm{sk}}^{\mathrm{C}}$ 的卷 积层 $\boldsymbol{\Phi}_{\mathrm{sk}}^{\mathrm{C}}$ 和一个参数为 $\boldsymbol{W}_{s k}^{C}, \boldsymbol{b}_{s k}^{C}$ 的全连接层组成。这 里的卷积层包括卷积操作、最大值池化 (第二层除外) 和 ReLU 激活函数。第 $k$ 个 CNN 学习器的输出如式 (2)所示。这些 CNN 学习器的参数都是通过混合多 任务损失函数来进行训练得到的, 因此可以比较灵 活地适应不同的任务

$$
\boldsymbol{x}_{s k}^{t}=\max \left\{\boldsymbol{W}_{s k}^{C} \Phi_{\mathrm{sk}}^{\mathrm{C}}\left(I_{s k}^{t}, \boldsymbol{\theta}_{\mathrm{sk}}^{\mathrm{C}}\right)+\boldsymbol{b}_{s k}^{\mathrm{C}}, \boldsymbol{0}\right\}
$$

\section{2 信息融合网络}

与人类视觉系统类似, 本方法将注意点位置信 息与表观特征融合到一起。先将多个尺度下的中央 凹描述子 $\left\{\boldsymbol{x}_{s k}^{t}\right\}$ 拼接到一起然后采用全连接网络将 其映射成表观特征描述子 $\boldsymbol{x}_{s}^{t}$ 。同时, 注意点坐标也 被映射成为一个与表观描述子同维度的矢量。

表观和位置融合: 有多种操作可以被用在全连 接层之前来进行表观和位置描述子的融合, 比如加
法 $\boldsymbol{\Phi}_{+}^{\mathrm{F}}$, 元素级乘法 $\boldsymbol{\Phi}_{\times}^{\mathrm{F}}$ 以及拼接 $\boldsymbol{\Phi}_{\mathrm{c}}^{\mathrm{F}}$ 等。融合之后 的描述子可以被表示为式(3)。其中, $\boldsymbol{\theta}^{\mathrm{F}}$ 是一个参数 集合，包括全连接层的权重和偏移。

$$
\boldsymbol{x}^{t}=\boldsymbol{\Phi}^{\mathrm{F}}\left(\boldsymbol{x}_{s}^{t}, \boldsymbol{x}_{P}^{t}, \boldsymbol{\theta}^{\mathrm{F}}\right) \boldsymbol{\Phi}^{\mathrm{F}} \in\left\{\boldsymbol{\Phi}_{+}^{\mathrm{F}}, \boldsymbol{\Phi}_{\times}^{\mathrm{F}}, \boldsymbol{\Phi}_{\mathrm{c}}^{\mathrm{F}}\right\}
$$

多注意定位点融合: 将 $T$ 个循环步骤中局部的 观测 $\left\{\boldsymbol{x}^{t}\right\}, t=1,2, \cdots, T$ 融合到一起是必要的。因此, $m$ 层的 LSTM 网络被用于学习这些观测 $\left\{\boldsymbol{x}^{t}\right\}$ 之间的 依赖关系和贡献情况, 这个过程与大脑皮层中记忆 一般性的规则相类似。最后一层 LSTM 的隐层状态 $\boldsymbol{h}_{\mathrm{m}}^{\mathrm{t}}$ 为作为整个 LSTM 映射 $\boldsymbol{\Phi}^{\mathrm{R}}$ 的最终输出。具体来 说, 多步注意融合过程可以被表示为

$$
\boldsymbol{h}_{m}^{t}=\boldsymbol{\Phi}^{\mathrm{R}}\left(\boldsymbol{h}^{t-1}, \boldsymbol{x}^{t}, \boldsymbol{\theta}^{\mathrm{R}}\right) t=1,2, \cdots, T
$$

式中, $\boldsymbol{\theta}^{\mathrm{R}}$ 是参数集合, $\boldsymbol{h}^{t-1}=\left\{\boldsymbol{h}_{1}^{t-1}, \boldsymbol{h}_{2}^{t-1}, \cdots, \boldsymbol{h}_{m}^{t-1}\right\}$ 是所 有 LSTM 层在上一个循环步骤的参数。与传统方 法 $^{[3]}$ 和 r-CNN ${ }^{[5]}$ 不同的是, 多注意融合提供了一种灵 活的方式从局部到全局来识别目标。

\section{3 多任务动作}

本网络的主要任务是定位、识别及预测下一个 注意点, 这些任务的输入都是多注意融合特征 $\boldsymbol{h}_{m}^{t}$ 。

定位和识别: 目标边框的几何参数 $O^{t}=\left[O_{y x}^{t}, O_{h w}^{t}, O_{s}^{t}\right]$ 包括左上点的位置, 目标大小以及 得分。它们是由参数为 $\boldsymbol{W}_{A}^{D}, \boldsymbol{b}_{A}^{D}$ 的全连接层回归得 到, 其后跟随一个超参数为 $\boldsymbol{\theta}_{\mathrm{c}}$ 的截断操作 $S_{c}$ 以保证 数值范围的合适。所有类别的概率是由参数为 $\boldsymbol{W}_{A}^{C}, \boldsymbol{b}_{A}^{C}$ 的全连接层和 Softmax 操作 $S_{f}$ 得到。具体来 看，该过程可以表示如下

$$
\begin{gathered}
O^{t}=S_{c}\left(\boldsymbol{W}_{A}^{D} \boldsymbol{h}_{m}^{t}+\boldsymbol{b}_{A}^{D}, \boldsymbol{\theta}_{\mathrm{c}}\right) \\
C^{t}=S_{f}\left(\boldsymbol{W}_{A}^{C} \boldsymbol{h}_{m}^{t}+\boldsymbol{b}_{A}^{C}\right)
\end{gathered}
$$

注意点预测: 本文从一个均值为 $\boldsymbol{\mu}_{P}^{t+1}$, 标准差 为 $\sigma$ 的高斯分布中采样下一个注意点。一个参数为 $\boldsymbol{W}_{A}^{P}, \boldsymbol{b}_{A}^{P}$ 的全连接层和 sigmoid 激活函数被用于估计 均值 $\boldsymbol{\mu}_{\mathrm{P}}^{t+1}$, 该过程可以表示如下

$$
\begin{gathered}
P_{f}^{t+1} \sim N\left(\boldsymbol{\mu}_{\mathrm{P}}^{t+1}, \sigma^{2}\right) \\
\boldsymbol{\mu}_{\mathrm{P}}^{t+1}=2 S\left(\boldsymbol{W}_{A}^{P} \boldsymbol{h}_{m}^{t}+\boldsymbol{b}_{A}^{P}\right)-1
\end{gathered}
$$

式中, $N(\cdot, \cdot)$ 表示高斯分布, $S(\cdot)$ 表示 sigmoid 函数。 多任务动作网络中的所有可变参数都是通过混合损 失函数端到端地训练得到。

\section{3 混合损失函数}

本文采用最小化损失函数 $L_{h}$ 的方式来优化整 个网络的参数。 $L_{h}$ 的各部分表示如下

$$
L_{h}=L_{d}+L_{\mathrm{c}}+L_{\mathrm{P}}
$$


式中, $L_{d}$ 是定位的损失函数, $L_{c}$ 是分类器的交叉熵, $L_{P}$ 表示注意点的损失函数。

定位损失: 本文假设一张图像 $I$ 只包含一个目 标, 其左上点坐标为 $O_{y x}^{g}$, 大小为 $O_{h w}^{g}$ 。最后一步对 于位置的预测量 $O_{y x}^{T}$ 被视为以真值为条件的随机变 量, 可以通过极大似然的方式进行估计。此外, 还 需要一个损失项来约束预测目标的大小 $O_{h w}^{T}$ 。本文 采用预测边框与真实边框的重叠率( IoU 映射)来构 造与预测大小相关的损失函数。试验上, 本文将重 叠率限制在区间 $\left[10^{-8}, 1\right]$ 内。因此定位任务的损失函 数 $L_{d}$ 表示如下

$$
\begin{gathered}
L_{d}=-\ln \left(P\left(O_{y x}^{T}\right)\right)- \\
\ln \left(\operatorname{IoU}\left(O_{h w}^{T}, O_{h w}^{g}, O_{y x}^{T}, O_{y x}^{g}\right)\right)
\end{gathered}
$$

位置预测的偏差量 $\left(O_{y x}^{T}-O_{y x}^{g}\right)$ 被选择为零均值的高 斯分布, 即

$$
O_{y x}^{T} \sim N\left(O_{y x}^{g}, \sigma_{O}^{2}\right)
$$

式中, $N(\cdot, \cdot)$ 表示高斯分布, 假设 $x$ 和 $y$ 坐标是相互 独立的, $\sigma_{O}$ 是标准差参数。

当预测边框与真实边框的重叠率为 0 的时候, 极大似然项会指导参数调整; 当重叠率不为 0 的时 候, 最大化 IoU 项能够改善预测边框的精度。

分类损失：本文采用交叉熵来测度在各个类别 上预测分布 $C^{T}$ 与真实分布 $C^{g}$ 的一致性。分类任务 的损失函数表示为 $L_{c}=\sum_{k=1}^{N}-C_{k}^{g} \ln \left(C_{k}^{T}\right)$, 其中 $N$ 是类 别的个数, $C_{k}^{g}, C_{k}^{T}$ 分别表示 $C^{g}$ 和 $C^{T}$ 的第 $k$ 个分量。

注意过程损失：除了检测和分类任务的损失函 数之外, 还需要与注意点相关的损失函数来约束注 意的预测和提取更有效的局部特征。

在增强学习中, 策略奖励机制被用于优化一个 决策过程。这里, 注意点的策略是基于最优均值下 的高斯分布。本文定义累计奖励 $R^{t}$ 为预测边框与真 实边框的重叠率。因此, 注意点的决策过程可以由 策略奖励 ${ }^{[25]}$ 的方法来进行优化, 其损失项表示如下

$$
L_{P}^{r}=-\sum_{t=1}^{T} \ln \left(\pi\left(P_{f}^{t} \mid \boldsymbol{\mu}_{\mathrm{P}}^{\mathrm{t}}, \sigma^{2}\right)\right)\left(R^{t}-b^{t}\right)
$$

式中, $\pi(\cdot, \cdot, \cdot)$ 是采样点 $P_{f}^{t}$ 的分布, $b^{t}$ 是 $R^{t}$ 的期望 ${ }^{[26]}$, 即 $b^{t}=E_{\pi}\left[R^{t}\right]$ 。最小化损失项 $L_{P}^{r}$ 可以减少梯度估计 的方差 ${ }^{[17]}$ 。

人类会将注意力集中在需要识别的目标上。为 了模拟这个机制, 本文采用 L2 损失来约束最后一 步注意点以使其接近目标的中心。因此, 关于注意 过程的整个损失函数如式(13)所示。式中, 得分 $O_{s}^{t}$ 在 $\mathrm{L} 2$ 准则下被优化来估计 $R^{t}$ 。整个的损失函数同时
包含随机的 $(\mathrm{S})$ 和目标启发 $(\mathrm{A})$ 的策略, 称为 SA 策 略。随机策略增大了样本的多样性, 有助于增强泛 化能力, 同时 $L_{P}^{r}$ 用来提高注意的效率。目标启发策 略使得最后一步注意点接近目标, 这样可以保证预 测和优化过程的稳定性。

$$
\begin{gathered}
L_{P}=L_{P}^{r}+\frac{1}{T} \sum_{t=1}^{T}\left(O_{s}^{t}-R^{t}\right)^{2}+ \\
\left\|\boldsymbol{\mu}_{\mathrm{P}}^{\mathrm{T}}-\left(O_{y x}^{g}+\frac{1}{2} O_{h w}^{g}\right)\right\|_{2}^{2}
\end{gathered}
$$

\section{4 试验}

\section{1 数据集}

本文的试验都是在三个数据集上进行的, 包括 两个 MNIST 数据集的变种和一个真实世界车辆检 测的数据集。

MSO 数据集和 MSNO 数据集: 本文建立了一个 新的数据集(MNIST scaled object, MSO), 在该数据集 中, 每个图像只包含一个具有边框和标记信息的数字 目标。MSO 数据集中的图像由两个步骤产生: (1) 从 $[0.3,1.5]$ 的均匀分布中采样一个随机的尺度对来改变 MNIST 数据集中的图像大小; (2) 上一步生成的图像 插入到一个 56 像素 $\times 56$ 像素的黑色背景中, 在随机 放置的同时保证不截断该目标。为了测试本方法对噪 声的敏感性, 本文还建立了另一个数据集(MNIST scaled and noised object, MSNO)。加入噪声的方式为向 $\mathrm{MSO}$ 数据集的每一个图像加入 6 个 6 像素 $\times 6$ 像素的图 像块, 这些图像块是从 MNIST 数据集中随机截取的。

FCAR 数据集: 特别地, 本文还建立了一个真 实世界的场景下用于车辆检测的数据集。在该数据 集中, 每幅图像可能包含不止一辆车, 但是感兴趣 的车辆只是与本车同车道的车辆。因此, 一幅图像 的标注信息就是被关注的车辆边框。数据集中的图 像是由安装在车辆上的固定前视摄像头获取的, 因 此包含更多的结构信息。这对于感兴趣的目标检测 是有意义的, 特别是对于小目标。在 FCAR 数据集 中, 训练集包含 6330 张图像, 测试集包含 2712 张图像, 分别率为 800 像素 $\times 800$ 像素。

\section{2 试验细节}

初始注意点: 初始的注意点是必须给出的参数。 本文将图像坐标转换到 $[-1,1]$, 然后将圆点设置为 图像的中心。初始注意点的选取就是在该坐标下, 从 $[-r, r], r \in[0,1]$ 的均匀分布中随机采样得到。

参数设置: 本网络的结构参数设置如表 1 所示。 中央凹描述子提取器的参数是根据输入图像大小调 整的, 所有 $\mathrm{CNN}$ 的结构是一致的, 参数稍有不同。 
表 1 各个子网络的结构参数的设置

\begin{tabular}{|c|c|c|c|c|}
\hline 子网络 & FDE & CNN 学习器 & IFN & MTA \\
\hline 参数 & $\begin{array}{c}s_{0}=8 \times 8 \\
s_{f}=2.0 \\
n=3 \\
s_{u}=2 s_{0}\end{array}$ & $\begin{array}{cc}\text { conv1+relu1: } & 5 \times 5 \times 1 \times 32 \\
\text { conv2+relu2: } & 5 \times 5 \times 32 \times 64 \\
\text { conv3+relu3: } & 5 \times 5 \times 64 \times 4 \\
\text { fc4+relu4: } & 1024 \times 256 \\
\text { softmax } & \end{array}$ & $\begin{array}{l}\text { scale fusion: } \\
\text { fc+relu: } 768 \times 256 \\
\text { location mapping: } \\
\text { fc+relu: } 2 \times 256 \\
\text { fusion method: } \boldsymbol{\Phi}_{+}^{\mathrm{F}} \\
\text { multi-fixation fusion: } \\
\text { LSTMs: }[256] \times 3\end{array}$ & $\begin{array}{c}\text { fc+clip: } 256 \times 5 \rightarrow\left\{O_{y x}^{t}, O_{h w}^{t}, O_{s}^{t}\right\} \\
\text { fc+softmax: } 256 \times 5 \rightarrow C^{t} \\
\text { fc+sigmoid }+N(\mu, \sigma): \\
256 \times 2 \rightarrow P_{f}^{t}, \sigma=0.2\end{array}$ \\
\hline
\end{tabular}

在处理 FCAR 数据集时, 本文设置 FDE 的参数 为 $s_{0}=32 \times 32, s_{f}=4.5, n=3, s_{u}=32 \times 32$, 第一个卷 积层的参数 $5 \times 5 \times 3 \times 32$, 并且移除分类动作, 其他 保持不变。

\section{3 结构参数的影响}

在 MSO 和 MSNO 数据集上, 本文采用四组试 验来探索所提出方法中结构参数的影响。这四组试 验的默认参数为

$$
r=0.3 \quad n=3 \quad T=10 \quad \boldsymbol{\Phi}^{F}=\boldsymbol{\Phi}_{+}^{F}
$$

每一组试验都只改变其中一个参数。

初始点范围: 由于具有不同的局部观测, 所以 随机选择初始点是增强和扩大数据集的一种方式。 本文依据均匀分布 $[-r, r]$ 来采样, 其中 $r \in\{0,0.3,0.5,0.7,0.9\}$ 。图 3a 展示了不同范围的 $\mathrm{mAP}$ 值。在没有随机性 $(r=0)$ 的情况下, 本方法在 MSNO 数据集上的精度比在 MSO 数据集上的精度 少 $19 \%$ 。这表明随机初始点对于获得在嘈杂背景下 的鲁棒性是有益的。

尺度个数: 多尺度能够提供更多的上下文信息, 这可以指导对下一个注意点的预测。本文在 1 到 3 的范围内改变尺度的个数来探索多尺度方法的影 响。图 3b 表明 3 尺度局部区域是一种比较好的获取 上下文的方法, 在 MSO 数据集上取得了 0.977 的 $\mathrm{mAP}$ 。

注意步数: 多步注意是另一种挖掘丰富上下文 线索的方式。本文在 $\{1,2,4,6,8,10\}$ 范围选择步数 $T$ 来进行试验。如图 $3 \mathrm{c}$ 所示, 随机一瞥 $(T=1)$ 是一个 不好的选择, 随着注意步数的增加, 结果逐渐变好。 $T=6$ 时在 MSO 数据集上可以取得 0.97 的 $\mathrm{mAP}$ 。可 以得出结论, 多步注意是一种改善性能的必须手段。

融合方法: 本文提供三种用于融合表观和位置 信息的方法, 包括相加 $(+)$, 相乘 $(\times)$ 和拼接 $(c)$ 。 图 3d 表明了这三种融合方法获得相似的 mAP 值, 在 MSO 数据集上是 0.97 , 在 MSNO 数据集上是 0.90。最终为了计算上的简洁起见, 本文选择相加 操作作为融合方法。

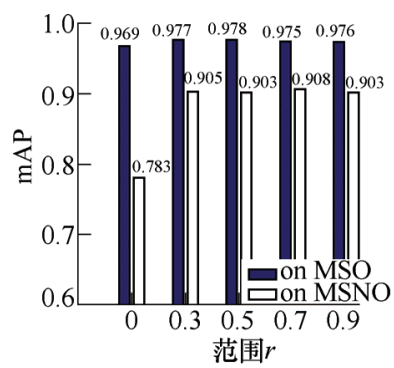

(a) 不同初始化范围 (b) 不同尺度个数

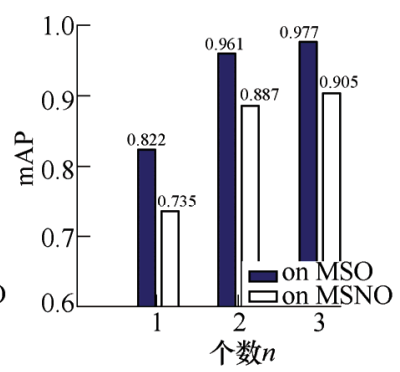

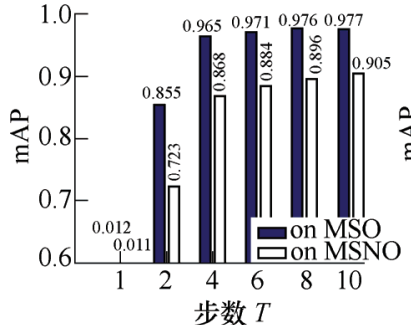

(c) 不同注意步数

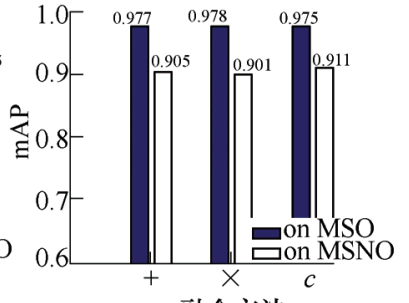

融合方法

(d) 不同融合方法
图 3 不同参数配置的比较

\section{4 注意点策略的意义}

本文设置了一些试验来分析随机策略和目标启 发策略的意义。对于随机策略 $(\mathrm{S})$ 在训练时采用 REINFORCE 规则的变种, 而在目标启发策略中没 有随机采样, 在训练时有 L2 损失约束。随机和目 标启发的混合方法(SA)也会在试验中进行考察。

在图 4 中展示了混合 SA 策略所产生的几个样 例, 在自动选择一个注意点序列(白色点)之后, 最 终预测结果(浅色方框)与真值(深色方框)是十分接 近的。从中可以发现，小目标和一些被污染的目标 也能被准确地检测和分类。
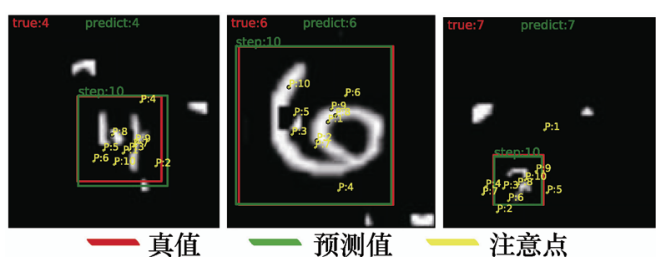

图 4 本方法在 MSNO 数据集上的典型结果实例

图 5a 表明 L2 损失能够有效指导目标启发策略中 的下一个注意点的预测，随机策略多余增强在嘈杂背 景下的鲁棒性是有益的。然而, 随机策略有可能会导 
致训练不收敛。混合的 SA 策略可以获得最高的 $0.905 \mathrm{mAP}$ ，同时取得收敛的稳定性。两种策略都不用 的方法(None)在 MSO 和 MSNO 数据集上都取得最差 的结果，这表明随机方法和 L2 损失的必要性。

表 2 中将本方法与其他方法进行了比较。在
CT100(cluttered translated $100 \times 100)^{[17]}$ 数据集上的 结果表明采用本文所提出的注意策略联合进行目标 检测定位是有益的。在 MSNO 数据集上, 本方法取 得最好的结果, 并且在检测分类嘈杂背景中的小目 标时具有优势。

表 2 本方法与其他方法在 MSNO 和 CT100 两个数据集上的比较

\begin{tabular}{lccccccc}
\hline \multicolumn{1}{c}{ 方法 } & \multicolumn{2}{c}{ RADCN(本文) } & LeNet+Regression ${ }^{[27]}$ & \multicolumn{2}{c}{ RAM $^{[17]}$} & \multicolumn{2}{c}{ DRAW $^{[23]}$} \\
\hline 数据集 & MSNO & CT100 & MSNO & MSNO & CT100 & MSNO & CT100 \\
平均 IoU & 0.879 & 0.918 & 0.643 & - & - & - & - \\
错误率(\%) & 6.8 & 3.72 & 42.1 & 19.7 & 8.11 & 19.5 & 3.36 \\
mAP & 0.905 & 0.940 & 0.395 & - & - & - & - \\
\hline
\end{tabular}

\section{5 真实图像的注意力目标检测}

在 FCAR 数据集上应用本方法来检测前车, 即 与本车在相同车道的车辆, 并且用平均 $\mathrm{IoU}$ 来进行 评价。对于训练过程, 由于该数据集没有类别信息, 分类的交叉熵损失函数被移除。

如图 5b 所示, 增加注意步数是一种简单有效改 善精度的方法。 (a) 不同注意策略的比较

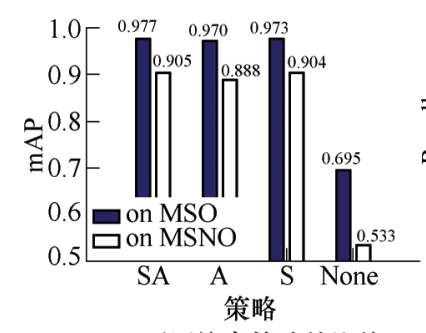

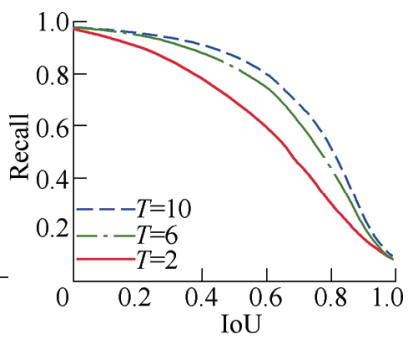

(b) 不同注意步数的比较
图 5 注意策略和注意步数对结果的影响

图 6 表明本方法对于比较小的和比较大的目 标都是有效的, 特别是, 能够忽略不同车道上那 些不感兴趣的车辆目标而只对前车感兴趣。预测 边框能够达到 0.70 的平均 $\mathrm{IoU}$ 。由于具有固定的 视角, 这些图像暗含了目标的分布信息。此外, 有一些上下文信息能够知道网络来定位前车, 比 如天空、地面、车道线和其他车辆等。因此, 本 方法能够灵活高效地学习有效特征, 挖掘丰富的 环境信息, 以及融合多步注意的局部观测来进行 感兴趣目标的搜索。
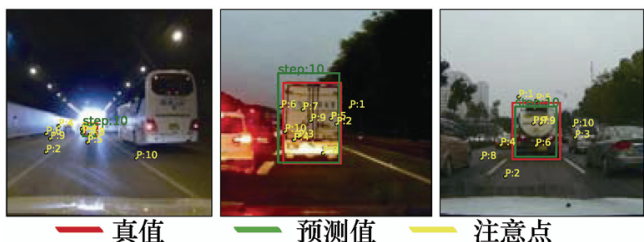

图 6 本方法在 FCAR 数据集上的典型结果实例

\section{6 运行效率分析}

与在全图进行卷积的方法相比较, 本方法通过
多个局部的多尺度局部区域是更加快速的。这些局 部区域被转换为 $m \times m$ 大小, 而全图的大小为 $M \times M, M \gg m$ 。那么, 在全图上进行卷积的次数为 $N_{g}=\frac{M^{2}}{n T m^{2}} N_{l}$, 其中 $n$ 是尺度的个数, $T$ 是注意的 步数, $N_{l}$ 是本方法在局部区域所用的卷积次数。在 FCAR 数据集上, $M=800, m=32, n=3, T=10$, 结 果是 $N_{\mathrm{g}} / N_{l}>20$, 这表明多步局部观测的方式所需 要的计算量要远低于在全图上进行观测所需的计算 量。本方法在 800 像素 $\times 800$ 像素的图像上进行 $T=10$ 的循环, 在 Tensorflow 软件平台和 M40 硬件平台上, 能够获得大约 30 帧/s 的速度。

\section{5 结论}

本文所提出的视觉注意网络与学习方法能够有 效地对感兴趣目标进行定位和识别。而且, 多任务 的混合损失函数能够用于对网络参数进行端到端地 训练。特别地, 随机注意和目标启发策略让网络更 加稳定和准确。在 FCAR 数据集上的试验表明, 本 方法能够利用有效的上下文信息来检测感兴趣的目 标, 特别是小目标。当前, 本模型在一幅图像中只 能检测一个目标。在将来的工作中, 该模型将会被 扩展到多目标的检测和分类。

\section{参 考 文 献}

[1] VIOLA P, JONES M J. Robust real-time face detection[J]. International Journal of Computer Vision，2004， 57(2): 137-154.

[2] FELZENSZWALB P F, GIRSHICK R B, MCALLESTER D A, et al. Object detection with discriminatively trained part-based models[J]. IEEE Transactions on Pattern Analysis and Machine Intelligence, 2010, 32(9) : 1627-1645

[3] SADEGHI M A, FORSYTH D. $30 \mathrm{~Hz}$ object detection 
with DPM V5[C]// European Conference on Computer Vision (ECCV). September 6-12, 2014, Zurich. Springer, 2014: 65-79.

[4] GIRSHICK R, DONAHUE J, DARRELL T, et al. Rich feature hierarchies for accurate object detection and semantic segmentation[C]// 2014 IEEE Conference on Computer Vision and Pattern Recognition (CVPR). June 23-28, 2014, Columbus, OH. IEEE, 2014: 580-587.

[5] GIRSHICK R. Fast R-CNN[C]// 2015 IEEE International Conference on Computer Vision (ICCV). December 7-13, 2015, Santiago, Chile. IEEE, 2016: 1440-1448.

[6] REN Shaoqing, HE Kaiming, GIRSHICK R, et al. Faster R-CNN: Towards real-time object detection with region proposal networks[J]. IEEE Transactions on Pattern Analysis and Machine Intelligence, 2015, 39(6) : 1137-1149.

[7] REDMON J, DIVVALA S K, GIRSHICK R B, et al. You only look once: Unified, real-time object detection[C]// 2016 IEEE Conference on Computer Vision and Pattern Recognition (CVPR). June 27-30, 2016, Las Vegas, NV, USA. IEEE， 2016: 779-788.

[8] LIU Wei, ANGUELOV D, ERHAN D, et al. SSD: Single shot multibox detector[C]// European Conference on Computer Vision (ECCV). October 8-16, 2016 , Amsterdam. Springer, 2016: 21-37.

[9] SCHMIDHUBER J, HUBER R. Learning to generate artificial FOVEA Trajectories for target detection[J]. International Journal of Neural Systems, 1991 , 02(01n02): 125-134.

[10] TORRALBAA, OLIVAA, CASTELHANO M S, et al. Contextual guidance of eye movements and attention in real-world scenes: The role of global features in object search[J]. Psychological Review, 2006, 113(4): 766-786.

[11] OLIVA A, TORRALBA A. The role of context in object recognition[J]. Trends in Cognitive Sciences, 2007, 11(12): 1-527.

[12] BELL S, ZITNICK C L, BALA K, et al. Inside-outside net: Detecting objects in context with skip pooling and recurrent neural networks[C]// 2016 IEEE Conference on Computer Vision and Pattern Recognition (CVPR). June 27-30, 2016, Las Vegas, NV, USA. IEEE, 2016: 2874-2883.

[13] HE Kaiming, ZHANG Xiangyu, REN Shaoqing, et al. Spatial pyramid pooling in deep convolutional networks for visual recognition[C]// European Conference on Computer Vision (ECCV), September 6-12, 2014, Zurich.
Springer, 2014: 346-361.

[14] LAROCHELLE H, HINTON G E. Learning to combine foveal glimpses with a third-order Boltzmann machine[J]. Advances in Neural Information Processing Systems (NIPS), 2010: 1243-1251.

[15] TANG Yichuan, SALAKHUTDINOV R. Learning Stochastic feedforward neural networks[J]. Advances in Neural Information Processing Systems, 2013, 1 : 530-538.

[16] REZENDE D J, MOHAMED S, WIERSTRA D, et al. Stochastic backpropagation and approximate inference in deep generative models[C]// International Conference on Machine Learning, June 21-26, 2014, Beijing. 2014: 1278-1286.

[17] MNIH V, HEESS N, GRAVES A, et al. Recurrent models of visual attention[J]. Advances in Neural Information Processing Systems, 2014, 1: 2204-2212.

[18] GRAVES A, WAYNE G, REYNOLDS M, et al. Hybrid computing using a neural network with dynamic external memory[J]. Nature, 2016, 538(7626): 471-476.

[19] RANZATO M. On learning where to look[J]. arXiv: Computer Vision and Pattern Recognition, 2014, 1: 1405.5488 .

[20] DENIL M, BAZZANI L, LAROCHELLE H, et al. Learning where to attend with deep architectures for image tracking $[\mathrm{J}]$. Neural Computation, 2012, 24(8): 2151-2184.

[21] XU K, BA J, KIROS R, et al. Show, attend and tell: Neural image caption generation with visual attention[C]// International Conference on Machine Learning, July 6-11, 2015, Lille. 2015: 2048-2057.

[22] BAZZANI L, LAROCHELLE H, MURINO V, et al. Learning attentional policies for tracking and recognition in video with deep networks[C]// International Conference on Machine Learning, June 28-July 2, 2011, Bellevue. 2011: 937-944.

[23] GREGOR K, DANIHELKA I, GRAVES A, et al. DRAW: A recurrent neural network for image generation[C]// International Conference on Machine Learning, July 6-11, 2015, Lille. 2015: 1462-1471.

[24] CAICEDO J C, LAZEBNIK S. Active object localization with deep reinforcement learning[C]// IEEE International Conference on Computer Vision (ICCV), December 7-13, 2015, Santiago, Chile. IEEE, 2016: 2488-2496.

[25] WILLIAMS R J. Simple statistical gradient-following algorithms for connectionist reinforcement learning $[\mathrm{J}]$. 
Machine Learning, 1992, 8(3): 229-256.

[26] SUTTON R S, MCALLESTER D A, SINGH S P, et al. Policy gradient methods for reinforcement learning with function approximation[J]. Advances in Neural Information Processing Systems, 1999, 1: 1057-1063.

[27] LECUN Y, BOSER B E, DENKER J S, et al. Backpropagation applied to handwritten zip code
recognition[J]. Neural Computation, 1989, 1(4): 541-551.

作者简介: 吕杰, 男, 1992 年出生。主要研究方向为计算机视觉。

E-mail: jiejielyu@outlook.com

罗芳颖, 女, 1996 年出生, 硕士研究生。主要研究方向为计算机视觉。 E-mail: fangying.luo@foxmail.com

袁泽剑(通信作者), 男, 1971 年出生, 副教授。主要研究方向为计算机 视觉与机器学习。

E-mail: yuan.ze.jian@xjtu.edu.cn 